\title{
Effect of Stretching Force on the Cells of Epithelial Rests of Malassez In Vitro
}

\author{
Teruyoshi Koshihara, ${ }^{1}$ Kenichi Matsuzaka, ${ }^{2}$ Toru Sato, ${ }^{1}$ and Takashi Inoue ${ }^{2}$ \\ ${ }^{1}$ Department of Crown \& Bridge Prosthodontics, Tokyo Dental College, 1-2-2, Masago, Mihama-ku, Chiba 261-8502, Japan \\ ${ }^{2}$ Oral Health Science Center hrc7, Department of Clinical Pathophysiology, Tokyo Dental College, 1-2-2, Masago, \\ Mihama-ku, Chiba 261-8502, Japan
}

Correspondence should be addressed to Kenichi Matsuzaka, matsuzak@tdc.ac.jp

Received 15 September 2009; Revised 28 December 2009; Accepted 8 February 2010

Academic Editor: Vincent Everts

Copyright ( $) 2010$ Teruyoshi Koshihara et al. This is an open access article distributed under the Creative Commons Attribution License, which permits unrestricted use, distribution, and reproduction in any medium, provided the original work is properly cited.

Background and Objective. The aim of this study was to investigate the behavior of cells from epithelial rest of Malassez (ERM) against stretching force. Material and Methods. ERM-cultured cells were stretched for 1 hour, at the cycle of 18\% elongation for 1 second followed by 1 -second relaxation. The cells without addition of stretching force were used as controls. The cells were observed by immunohistochmical staining using actin 0, 12, 24, 36, 48, and 72 hours. Furthermore, expressions of HSP70-, VEGF-, and OPN-mRNAs of cells were also evaluated using quantitative RT-PCR. Results. Actin filaments were randomly orientated in the cytoplasm in the control group, whereas in the stretching group, actin filaments were orientated comparatively parallel to the stretching direction. Expression of HSP70-mRNA in the stretching group was significantly higher than that of control group at 12 , 24,36 hours $(P<.05)$. Expression of VEGF-mRNA in the stretching group was significantly higher than that of control group at $24,36,48$, and 72 hours $(P<.05)$. Expression of OPN-mRNA in the stretching group was significantly higher than that of control group at 12 and 24 hours $(P<.05)$. Conclusion. ERM cells response against the stretching force by expressing HSP70, VEGF, and OPN.

\section{Introduction}

Epithelial-mesenchymal interactions play important roles during tooth formation in the jaw. After the crown formation, the inner and outer enamel epithelium joins together and begins to migrate towards the apical area to form the tooth root. This epithelium is called Hertwig's epithelial root sheath (HERS). HERS cells induce dental papilla cells to differentiate into odontoblasts to form the root dentin and they begin to fragment just before cementogenesis.

After fragmentation of the HERS, some epithelial cells move away from the root surface and remain throughout life in the periodontal ligament. Those cell islands are called epithelial rests of Malassez (ERM) [1-3]. It has been reported that the functions of the ERM are to regulate the width of the periodontal ligament space $[4,5]$, the growth induction of nerve endings [6], the differentiation of ameloblasts [7], the secretion of enamel protein [8], and the differentiation induction of cementoblasts [5,9]. Furthermore, the ERM is known to respond to inflammatory cytokines [10], and this may induce increased numbers of cells of the ERM and create either a pocket epithelium [11] or the lining epithelium of the radicular cyst. Moreover, sometimes this process is related to the formation of odontogenic tumors, such as ameloblastomas or odontogenic carcinomas $[7,11,12]$.

The periodontal ligament is always exposed to mechanical stress such as compression forces or extension forces during occlusion and chewing. Those mechanical stresses are much milder compared to bridges or denture supported teeth. However, it is known that the periodontal space is kept fixed by instructions from stress immunity factors such as heat shock proteins (HSPs) except in the case of excessive power $[13,14]$.

It is known that small amounts of HSP exist constantly in all cells, and levels of HSP increase following chemical or mechanical stimulations such as heat [15], oxidative 
stress $[16,17]$, acid [18], ischemia, or reperfusion in situ [19]. When a tissue is subjected to stress as described above, HSP initiates biophylactic reactions and acts intracellularly as molecular chaperones and to maintain tissue homeostasis.

Vascular endothelial growth factor (VEGF) is an essential mediator for angiogenesis [20]. VEGF is produced by many kinds of cells during the vascularization process of developing embryos [21] and during the pathophysiologic processes of ischemia or wound healing $[22,23]$.

Osteopontin (OPN) is a bone-related protein involved in the production of tooth enamel, dentin, and the cementum. However, it is known that OPN has various other functions elsewhere. For these reasons, HSP, VEGF, and OPN are used as markers for cell responses against biological stress [24].

Morphological molecular studies [25-27] have characterized processes when stretching forces are loaded on periodontal ligament fibroblasts in vivo and in vitro. However, only a few morphological studies have examined the effect of stretching forces on cells of the ERM [28], and no molecular studies have characterized that.

The purpose of this study was to investigate the behavior of cells derived from the ERM against stretching forces in vitro, and to investigate them at the molecular level.

\section{Materials and Methods}

2.1. Cell Culture. Porcine ERM cells were provided by Prof. Abiko, Department of Dental Science, Institute of Personalized Medical Science, Health Sciences University of Hokkaido. ERM cells were cultured in $75 \mathrm{~cm}^{2}$ tissue culture flasks (Corning, Tokyo, Japan) with $\alpha$-MEM (Gibco, Carlsbad, CA, USA) containing 10\% fetal bovine serum (Sigma, St. Louis, MO, USA) and gentamycin (Sigma). They were cultured by incubation in a humidified atmosphere of $95 \%$ air and $5 \% \mathrm{CO}_{2}$ at $37^{\circ} \mathrm{C}$. After the cells became confluent, they were detached using trypsin/ethylenediaminetetraacetic acid (EDTA) (0.25\% w/v trypsin/0.02\% EDTA, pH 7.2). Subsequently, the cells were resuspended in the supplemented culture medium as described above and used in the following experiments. To identify epithelial cells, Immunofluorescence staining was carried out using a primary antibody against cytokeratin 19 (1:100 dilution; Abcam, Cambridge, UK) and a secondary antibody labeled with FITC (1:100 dilution; Invitrogen, Carlsbad, CA, USA) and were observed using a laser scanning microscope (LSM5 DUO, Carl Zeiss, Oberkochen, Germany).

2.2. Mechanical Stretching. Approximately $5.0 \times 10^{4} \mathrm{ERM}$ cells were seeded in flexible-bottomed culture plates coated with type I collagen (BioFlex Collagen; Flexcell International, McKeesport, PA, USA) and were cultured. After 1 week of culture, the ERM cells were stretched for 1 hour, with a cycle of $18 \%$ elongation for 1 second followed by 1-second relaxation, using an FX-4000 Flexercell Strain Unit (Flexcell International). The flexible cell-covered elastomer membranes were stretched by applying an oscillating vacuum to the

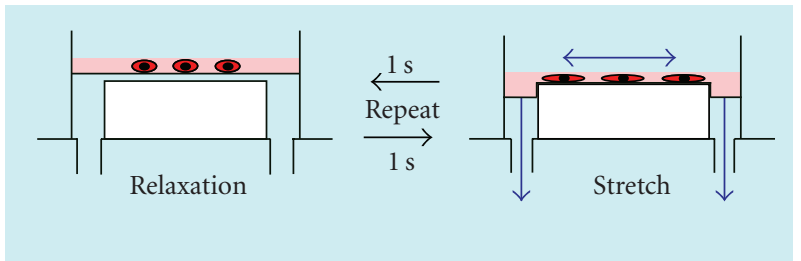

FIGURE 1: Stretching force was added continued for 1 hour at the cycle of $18 \%$ elongation for 1 second followed by 1 -second relaxation.

underside of the membranes and the duration, amplitude, and frequency of the stretch applied were controlled by a computer (Figure 1). The vacuum manifold that held the plates was maintained at $37^{\circ} \mathrm{C}$ in a humidified incubator with $95 \%$ air and $5 \% \mathrm{CO}_{2}$. The cells were collected at $0,12,24,36$, 48, and 72 hours after the initiation of the stretching force (stretching group). Cells without the stretching force were used as controls (control group).

Cell numbers were counted using a coulter counter (Beckman Coulter, Tokyo, Japan) at each of the time periods.

2.3. Immunofluorescence Observation. ERM cells were observed every day of the experiments using an ECLIPSE TS100 phase microscope (Nikon, Tokyo, Japan).

For standard fluorescence microscopic observations, cells in the dishes were washed with PBS, fixed with $4 \%$ paraformaldehyde for 20 minutes at room temperature and then were permeabilized in $0.1 \%$ Triton X-100 solution for 10 minutes. The cells were then washed twice with PBS. Nonspecific binding was blocked with 1\% BSA. Filamentous actin (F-actin) stress fibers were visualized using FITCconjugated phalloidin (1:100; Molecular Probes, Carlsbad) and were observed using a laser scanning microscope (LSM5 DUO, Carl Zeiss, Oberkochen ).

For investigating the effects of mechanical stretching in terms of protein levels, Immunofluorescence staining was carried out using a primary antibody against HSP70 ( $1: 200$ dilution; Abcam, Cambridge, UK) for 24 hours control and stretch group, VEGF (1:50 dilution; Abcam) for 48 hours control and stretch group, and OPN (1:40 dilution; Abcam) for 24 hours control and stretch group, a secondary antibody labeled with rhodamine (1 : 100 dilution; Invitrogen, Carlsbad, CA, USA) and were observed using a laser scanning microscope.

2.4. Actin Filament Direction Analysis. The line was drawn from center point to the periphery of the culture dish through the center of cell nucleus in both groups and the angle made by this line and each of the actin filament in the cytoplasm on the Immunofluorescence picture was measured as actin filament direction (Figure 2). For quantification, each angle created by actin filament against the line in the 5 cells on each of 5 culture dish in the both groups was analyzed using Image J analysis software (NIH, Bethesda, MD, USA). 
TABle 1: Primer sequence.

\begin{tabular}{lccc}
\hline & & Sequence & Product Size \\
\hline \multirow{2}{*}{ Hsp70 } & Forward: & $5^{\prime}$-CGGACGAGTACAAGGTTGA-3' & 206 \\
& Reverse: & $5^{\prime}$-CTCTTTCTCCGCCAACTG-3' & 154 \\
\multirow{2}{*}{ VEGF } & Forward: & $5^{\prime}$-TTCCGAGAGTACCCCGATGA-3' & 248 \\
& Reverse: & $5^{\prime}$-GGTGAGGTTTGATCCGCATA-3' & \\
\hline \multirow{2}{*}{ OPN } & Forward: & $5^{\prime}$-ACCGATCCGACGAGTCTCA-3' & 195 \\
& Reverse: & $5^{\prime}$-GGTACCATCCGTCTCCTCACTT-3' \\
\hline \multirow{2}{*}{ GAPDH } & Forward: & $5^{\prime}$-AGGGGCTCTCCAGAACATCA-3' & \\
\hline
\end{tabular}

2.5. $m R N A$ Expression Analyses. In order to investigate the effects of mechanical stretching in terms of mRNA levels, quantitative real-time reverse transcriptase-polymerase chain reaction (RT-PCR) using a LightCycler was employed. Total RNA was extracted from each sample using the acid guanidinum thiocyanate/phenol-chloroform method. Cultured cells at each of the time periods were homogenized using a Bransonic sonicator (Branson, Danbury, CT, USA) and were solubilized in TRIzol Reagent (Invitrogen, Tokyo, Japan) and chloroform. Supernatants were obtained by centrifugation at $13200 \mathrm{rpm}$ for 20 minutes at $4^{\circ} \mathrm{C}$, added to isopropanol, stored for over 1 hour at $-80^{\circ} \mathrm{C}$, and were finally centrifuged at $13200 \mathrm{rpm}$ for 20 minutes at $4^{\circ} \mathrm{C}$. The precipitates were obtained by decantation and were washed with $70 \%$ ethanol. The RNA pellets were dissolved in RNAase-free water and kept at $-20^{\circ} \mathrm{C}$ until use. Total RNA concentrations were measured by absorbance using a Nanodrop (ND-1000 Spectrophotometer, Scrum, Tokyo, JAPAN). Total RNA $(1 \mu \mathrm{g})$ was reverse transcribed and amplified in $20 \mu \mathrm{L}$ using an RT-kit (QIAGEN, MD, USA). cDNA synthesis was carried out using a Thermal Cycler (ThermoHybrid; ThermoBioAnalysis, Yokohama, Japan), and samples were incubated at $42^{\circ} \mathrm{C}$ for 15 minutes, heated to $99^{\circ} \mathrm{C}$, and then quick-chilled to $5^{\circ} \mathrm{C}$. Quantitative RT-PCR assays were performed using a LightCycler (Roche Molecular Biochemicals, Tokyo, Japan). Each reaction mixture considered $2 \mu \mathrm{L}$ LightCycler-FastStart DNA Master SYBR Green, $0.5 \mu \mathrm{M}$ of each primer, $5 \mathrm{mM} \mathrm{MgCl}_{2}$, and $2 \mu \mathrm{L}$ samples cDNA. Samples (total $20 \mu \mathrm{L}$ volume) were infused into glass capillaries. PCR was then carried out and mRNA expression of HSP70, VEGF, OPN, and the housekeeping gene GAPDH was measured using a LightCycler (Roche Diagnostics K.K.). The primer sequences for HSP70, VEGF, OPN, and GAPDH are shown in Table 1. The ratios of HSP70, VEGF, and OPN mRNAs were normalized against GAPDH. PCR data are reported compared to the corresponding control at 0 hour.

2.6. Statistical Analysis. Data from each experiment were statistically analyzed, and the assumption of homogeneity of variances and normal distribution of errors were tested for the response variables evaluated. Student's $t$-test was used to compare the actin filament direction and number of cells. One-way analysis of variance (ANOVA) and multiple comparison test (Bonfferroni's test) were used to compare

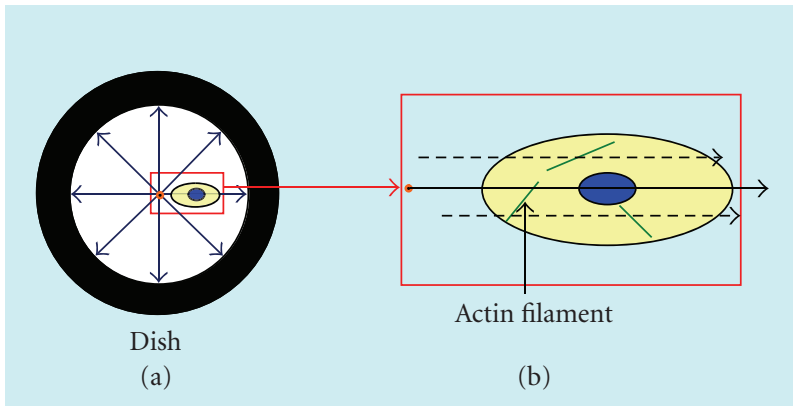

FIGURE 2: Stretching force is loaded equally by stretching machine (a). Stretching line was drawn from center point to the periphery of the culture dish through the center of cell nucleus in both groups. The angle made by this line and each of the actin filament in the cytoplasm on the Immunofluorescence picture was measured (b).

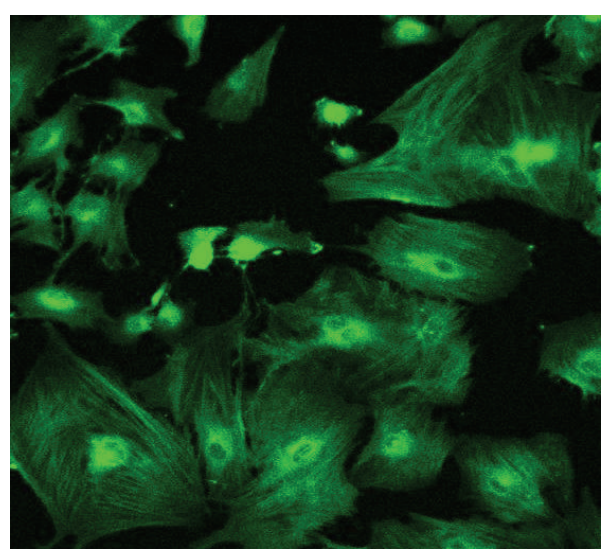

FIGURE 3: Immunofluorescence image of ERM cells using an antibody to cytokeratin 19 (CK 19). ERM cells were positive for CK 19.

mRNA levels for the control and stretching groups, and Student's $t$-test was used to compare various time periods $(P=.05)$.

\section{Results}

3.1. Immunofluorescence Microscopic Observations of CK 19. MER cells were stained for cytokeratin 19 using an immunofluorescence technique (Figure 3). 


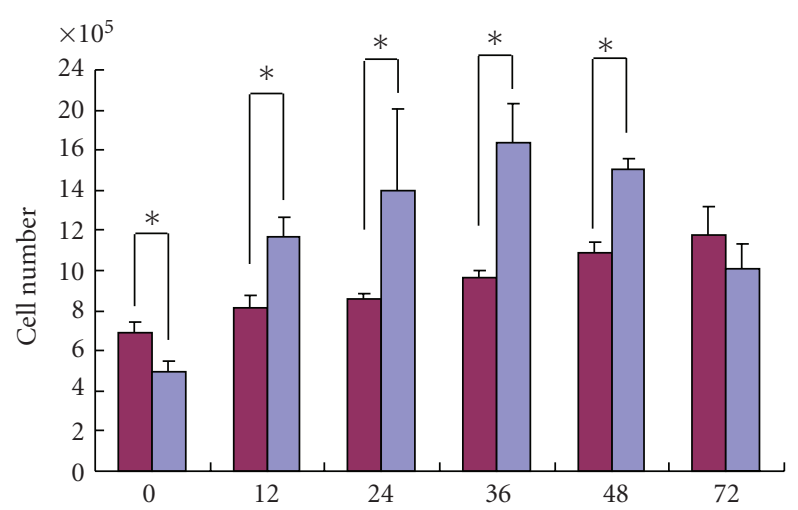

(h)

$$
\begin{aligned}
& { }^{*} P<.05 \\
& \square \text { Control } \\
& \square \text { Stretch }
\end{aligned}
$$

Figure 4: The numbers of cells in the stretching group were significantly less than the control group at 0 hour but were increased compared to the control group at 12, 24, 36, and 48 hours.

3.2. Cell Proliferation Ratio. The numbers of cells in the stretching group were significantly less than in the control group at 0 hour. However, the numbers of cells in the stretching group were significantly increased compared to the control group at 12, 24, 36, and 48 hours (Figure 4).

\subsection{Phase and Immunofluorescence Microscopic Observations} and Actin Filament Direction. There were no remarkable morphological changes that could be seen between the control group and the stretching group at 0 hour (Figure 5).

Actin filaments were randomly oriented in the cytoplasm of the control group $(43.9 \pm 5.2$ degree $)$, whereas in the stretching group, actin filaments were oriented parallel to the stretching direction $(23.4 \pm 1.9$ degree) (Figures 6 and 7). There was a significant difference between the control groups and the test groups in terms of actin filament direction $(P<.05)$.

\subsection{Expression of HSP70-, VEGF-, and OPN-mRNAs}

3.4.1. HSP70-mRNA. The expression of HSP70-mRNA in the stretching group was significantly higher than that in the control group at 12, 24, and 36 hours $(P<.05)$. However there was no significant difference at 48 or 72 hours. The expression of HSP70-mRNA in the stretching group showed the highest value at 24 hours and decreased with time (Figure 8).

3.4.2. VEGF-mRNA. The expression of VEGF-mRNA in the stretching group was significantly higher than that in the control group at 36, 48, and 72 hours $(P<.05)$. The expression of VEGF-mRNA in the stretching group showed the highest values at 48 hours and decreased with time (Figure 9).

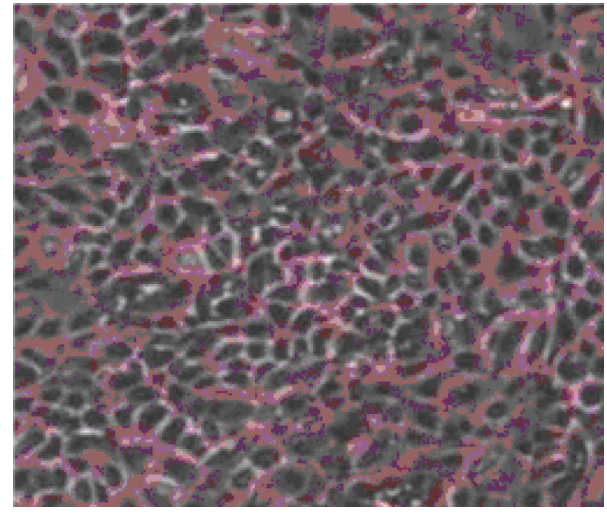

Control

(a)

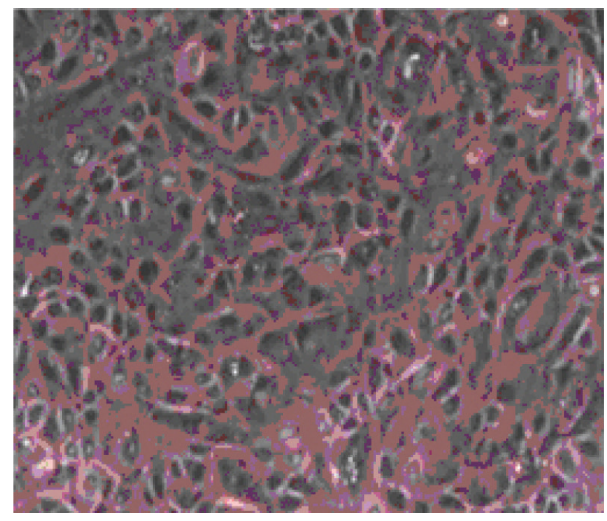

Stretch

(b)

FIGURE 5: There were no remarkable morphological changes that could be seen between the control group and the stretching group at 0 hour.

3.4.3. OPN-mRNA. The expression of OPN-mRNA in the stretching group was significantly higher than that in the control group at 12, 24, and 36 hours $(P<.05)$. The expression of OPN-mRNA in the stretching group showed the highest value at 24 hours (Figure 10).

The expression of HSP70-, VEGF-, and OPN-mRNAs was not significantly different in the control groups at any of the time periods.

3.4.4. Immunofluorescence Assay for Protein Level of HSP70, $V E G F$, and OPN. MER cells were immunoreacted byr HSP70 at 24 hours stretch group (Figure 11), VEGF at 48 hours stretch group (Figure 12), and OPN at 24 hours stretch group (Figure 13).

\section{Discussion}

The Flexercell system used in this study assigned a specific stretching force to the cultured cells. Using this system, we applied intermittent mechanical stretching to produce $18 \%$ elongation at the periphery of each well and that 


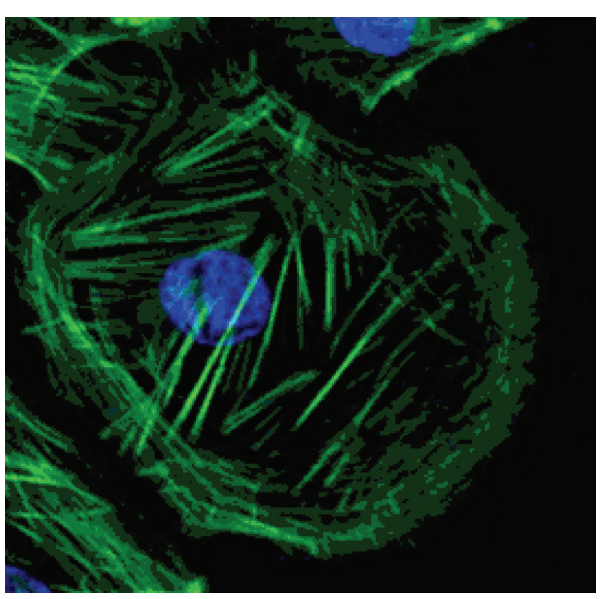

Control

(a)

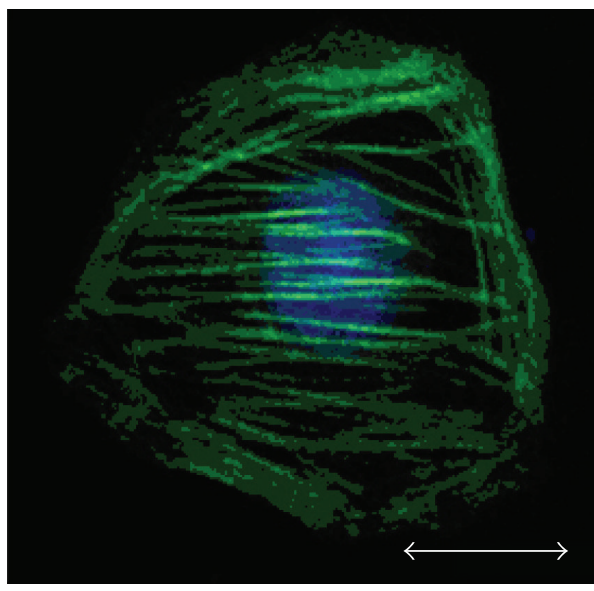

Stretch

(b)

FIGURE 6: Actin filament directions of the cells in stretching groups were significantly lower degree than those of control groups $(P<$ $.05)$.

elongated each cell body equally approximately $18 \%$. This force is almost identical to those used in previous studies in which $18 \%$ cell stretching is a slightly higher level than the physiological force reported earlier [25].

In this study, we found that the direction of actin filaments in the experimental groups immediately after the stretching force was arranged depending on the attractive direction, while the filaments of the control groups were arranged randomly. However, Buck [29] and Dartsch [30] reported that the direction of actin filaments was arranged at a right angle to the stretching direction after 24 hours of stretching force and they concluded that the cells might be reacting against the stretching force. Most probably, the cells initially react to the stretching force after which they recover naturally.

Actin filaments are known to combine with HSP70 constantly [31], and it is thought that cytoskeleton proteins and stress proteins interact in stressed cells [32]. For

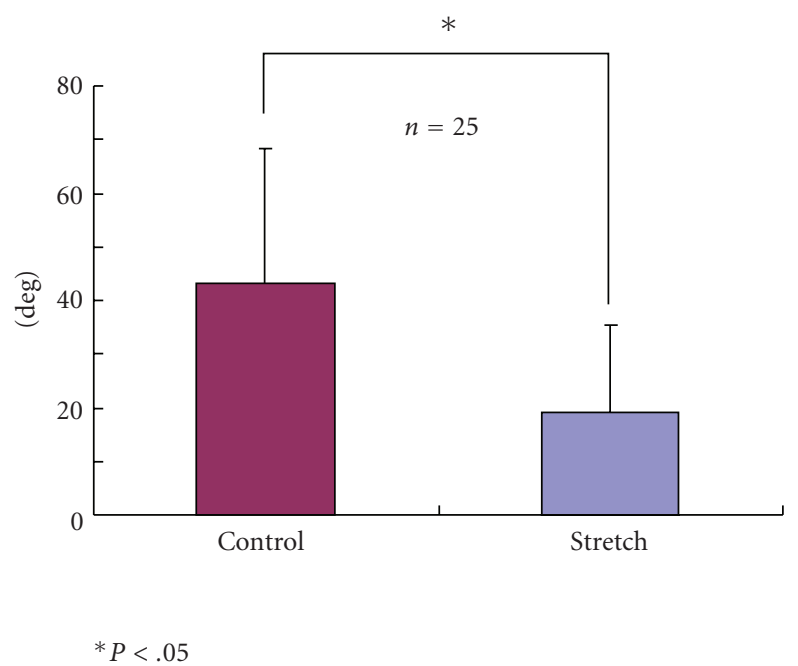

FIGURE 7: Actin filament directions of the cells in stretching groups were significantly lower degree than those of control groups $(P<$ $.05)$.

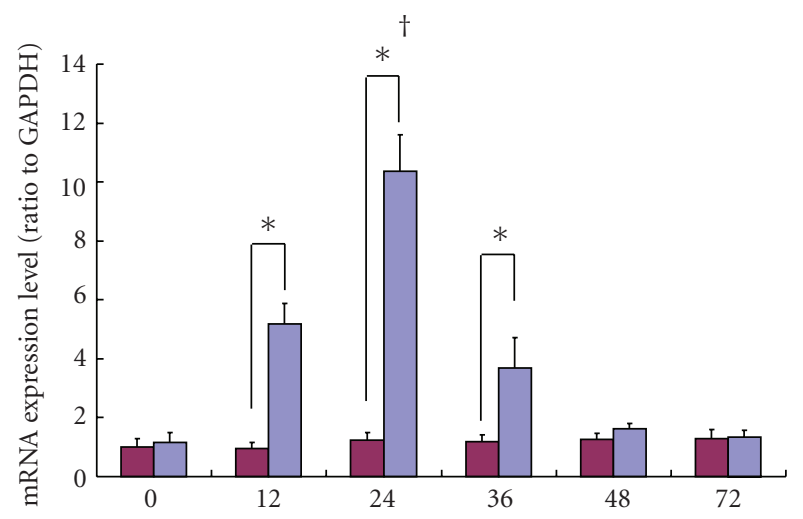

(h)

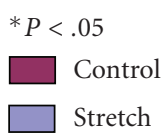

FIGURE 8: The expression of HSP70-mRNA in the stretching group was significantly higher than in the control group at 12,24 , and 36 hours $(P<.05)$. The expression of HSP70-mRNA in the stretching group showed the highest value at 24 hours and decreased with time (†).

this reason, it is thought that HSP70 may transmit the information in cooperation with integrins on the cell surface and cytoskeleton proteins when stretching forces are loaded.

In the stretching groups, more cell proliferation was observed than in the control groups. Brunette reported that mechanical stretching increases the number of epithelial cells synthesizing DNA [28]. Peake reported that deformed actin filaments promote cell proliferation [33]. Our study supports three reports and suggests that resistance of the ERM to the stretching stress may be high in the same way as fibroblasts [34]. 


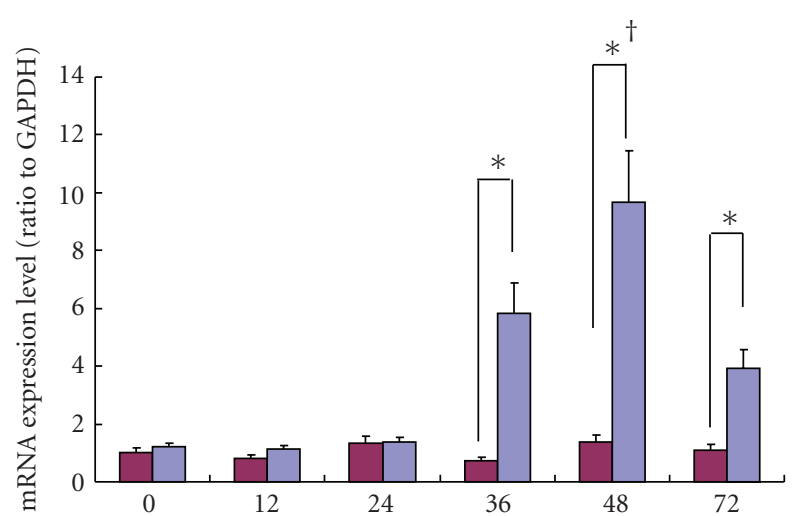

(h)

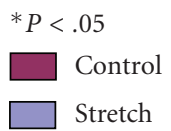

FIGURE 9: The expression of VEGF-mRNA in the stretching group was significantly higher than that is the control group at $24,36,48$, and 72 hours $(P<.05)$. The expression of VEGF-mRNA in the stretching group showed the highest value at 48 hours and decreased with time $(\dagger)$.

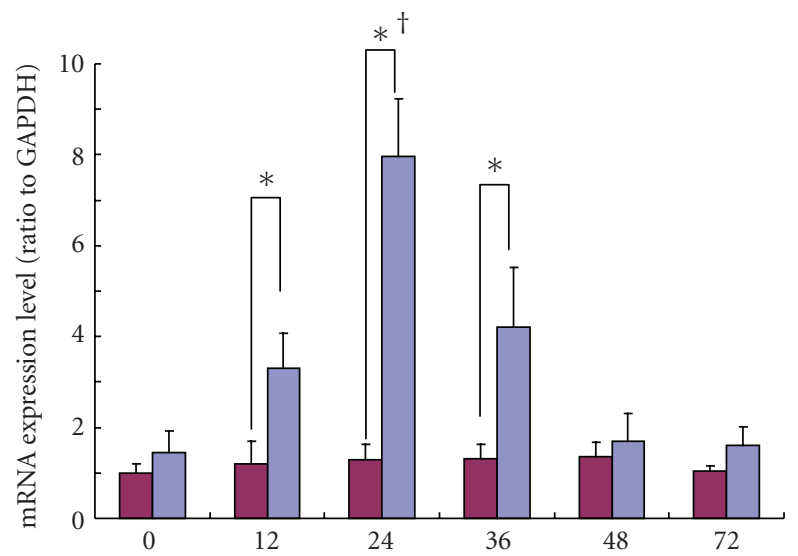

(h)

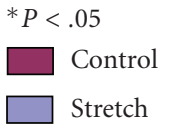

FIGURE 10: The expression of OPN-mRNA in the stretching group was significantly higher than the control group at 12 and 24 hours $(P<.05)$. The expression of OPN-mRNA in the stretching group showed the highest value at 24 hours $(t)$.

Beere and coworkers reported that HSP expresses by a steady state combines with other proteins such as actin filaments $[35,36]$. So, HSP guided by stress is thought to be important to the resistance of cells. In this study, the expression of HSP70 mRNA increased in the stretching group at 12, 24, and 36 hours, but not in the control group. This suggests that HSP70 probably reacts as autocrine to the damaged cells and maintains homeostasis of cells oneself.

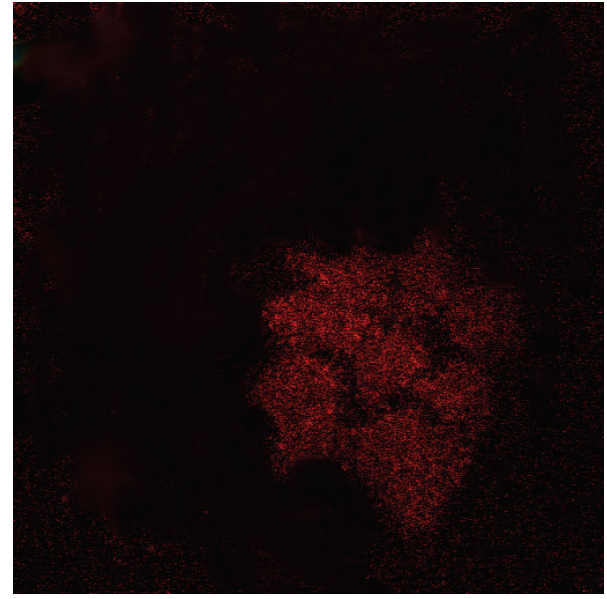

FIGURE 11: Immunofluorescence image of ERM cells using an antibody to HSP70 at 24 hours. ERM cells were positive for HSP70 at 24 hours stretch group.

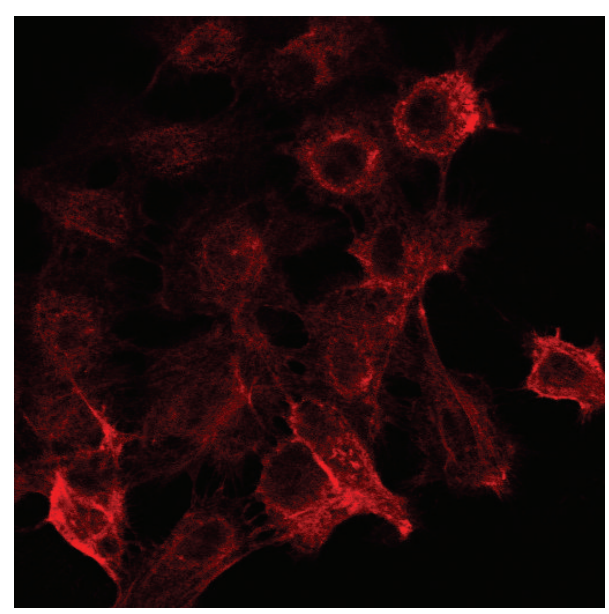

FIGURE 12: Immunofluorescence image of ERM cells using an antibody to VEGF at 48 hours. ERM cells were positive for VEGF at 48 hours stretch group.

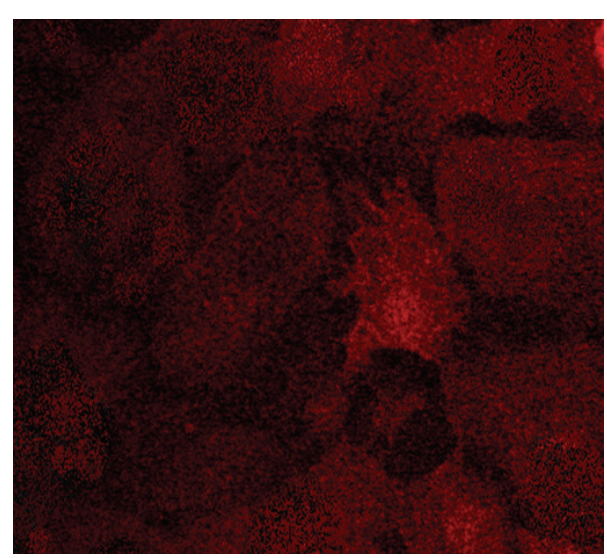

FIGURE 13: Immunofluorescence image of ERM cells using an antibody to OPN at 24 hours. ERM cells were positive for OPN at 24 hours stretch group. 
It has been reported that VEGF resists the stresses of compression [37], hypoxia, and reoxygenation [19] and restores and maintains the increased activity of cells in the tissues. The expression of VEGF suggests that the cells have an important role in the homeostasis of the tissue in terms of nutrition. Ohshima et al. reported that ERM cells express VEGF, but periodontal ligament fibroblasts express VEGF in vitro [38]. In this study, the expression of VEGF was not detected in the control group; however, increased expression of VEGF at 24, 36, 48, and 72 hours was noted in the stretching group. As a result, the homeostasis of the periodontal ligament might be maintained by VEGF secreted by cells of the MER under conditions of stretching as paracrine.

OPN has been known as a marker for osteogenesis and differentiation of bone cells. ERM cells are also known to produce OPN in conjunction with cementogenesis and bone formation $[39,40]$. Furthermore, it has been reported that periodontal ligament cells produce OPN under conditions of stretching. In addition, OPN is related to the increased activity of VEGF [41]. In this study, the expression of OPN was not remarkably changed in the control group; however, there was a significant increase in OPN mRNA at all time periods in the stretching group. The expression of OPN mRNA may control the hard tissue formation in the periodontal ligament. From these results, the expression of VEGF may occur first after which OPN expresses. This suggests that the ERM most probably maintains cementogenesis and osteogenesis against mechanical stress by expression of OPN.

\section{Conclusion}

ERM cells response against the stretching force by expressing HSP70, VEGF, and OPN.

\section{Acknowledgments}

The authors would like to thank Ms. Saori Takano and members of the Department of Clinical Pathophysiology, Tokyo Dental College, for their technical assistance. This research was supported in part by Oral Health Science Center Grant hrc7 from the Tokyo Dental College by a "High-Tech Research Center" Project for Private Universities: matching fund subsidy from the Ministry of Education, Culture, Sports, Science and Technology, Japan, 2006-2010, and 2007-2010 (no. 19592414).

\section{References}

[1] J. C. Rincon, W. G. Young, and P. M. Bartold, "The epithelial cell rests of Malassez-a role in periodontal regeneration?" Journal of Periodontal Research, vol. 41, no. 4, pp. 245-252, 2006.

[2] Y. Hamamoto, T. Nakajima, and H. Ozawa, "Ultrastructural and histochemical study on the morphogenesis of epithelial rests of Malassez," Archives of Histology and Cytology, vol. 52, no. 1, pp. 61-70, 1989.
[3] Y. Hamamoto, T. Nakajima, and H. Ozawa, "Ultrastructure of epithelial rests of Malassez in human periodontal ligament," Archives of Oral Biology, vol. 34, no. 3, pp. 179-185, 1989.

[4] J. D. Spouge, "A new look at the rests of Malassez. A review of their embryological origin, anatomy, and possible role in periodontal health and disease," Journal of Periodontology, vol. 51, no. 8, pp. 437-444, 1980.

[5] M. Shimono, T. Ishikawa, H. Ishikawa, et al., "Regulatory mechanisms of periodontal regeneration," Microscopy Research and Technique, vol. 60, no. 5, pp. 491-502, 2003.

[6] I. Lambrichts, J. Creemers, and D. Van Steenberghe, "Periodontal neural endings intimately relate to epithelial rests of Malassez in humans: a light and electron microscope study," Journal of Anatomy, vol. 182, no. 2, pp. 153-162, 1993.

[7] Y. Hamamoto, N. Hamamoto, T. Nakajima, and H. Ozawa, "Morphological changes of epithelial rests of Malassez in rat molars induced by local administration of $\mathrm{N}$ methylnitrosourea," Archives of Oral Biology, vol. 43, no. 11, pp. 899-906, 1998.

[8] Y. Hamamoto, T. Nakajima, H. Ozawa, and T. Uchida, "Production of amelogenin by enamel epithelium of Hertwig's root sheath," Oral Surgery, Oral Medicine, Oral Pathology, vol. 81, no. 6, pp. 703-709, 1996.

[9] N. Hasegawa, H. Kawaguchi, T. Ogawa, T. Uchida, and H. Kurihara, "Immunohistochemical characteristics of epithelial cell rests of Malassez during cementum repair," Journal of Periodontal Research, vol. 38, no. 1, pp. 51-56, 2003.

[10] J. D. Spouge, "A study of epithelial odontogenic residues in the pig," Journal of Periodontology, vol. 57, no. 3, pp. 164-171, 1986.

[11] A. R. Ten Cate, "The epithelial cell rests of Malassez and the genesis of the dental cyst," Oral Surgery, Oral Medicine, Oral Pathology, vol. 34, no. 6, pp. 956-964, 1972.

[12] D. M. Brunette, J. N. M. Heersche, A. D. Purdon, J. Sodek, H. K. Moe, and J. N. Assuras, "In-vitro cultural parameters and protein and prostaglandin secretion of epithelial cells derived from porcine rests of Malassez," Archives of Oral Biology, vol. 24, no. 3, pp. 199-203, 1979.

[13] S. Kalia, B. Melsen, and C. Verna, "Tissue reaction to orthodontic tooth movement in acute and chronic corticosteroid treatment," Orthodontics \& Craniofacial Research, vol. 7, no. 1, pp. 26-34, 2004.

[14] C. G. Walker, Y. Ito, S. Dangaria, X. Luan, and T. G. H. Diekwisch, "RANKL, osteopontin, and osteoclast homeostasis in a hyperocclusion mouse model," European Journal of Oral Sciences, vol. 116, no. 4, pp. 312-318, 2008.

[15] N. Fan, G. S. Yang, J. H. Lu, N. Yang, and H. B. Zhang, “Oral administration of geranylgeranylacetone plus local somatothermal stimulation: a simple, effective, safe and operable preconditioning combination for conferring tolerance against ischemia-reperfusion injury in rat livers," World Journal of Gastroenterology, vol. 11, no. 36, pp. 5725-5731, 2005.

[16] T. Ohkawara, H. Takeda, M. Nishiwaki, J. Nishihira, and M. Asaka, "Protective effects of heat shock protein 70 induced by geranylgeranylacetone on oxidative injury in rat intestinal epithelial cells," Scandinavian Journal of Gastroenterology, vol. 41, no. 3, pp. 312-317, 2006.

[17] I. S. Reus, I. Bando, D. Andres, and M. Cascales, "Relationship between expression of HSP70 and metallothionein and oxidative stress during mercury chloride induced acute liver injury in rats," Journal of Biochemical and Molecular Toxicology, vol. 17, no. 3, pp. 161-168, 2003. 
[18] L. Bardella and R. Comolli, "Differential expression of c-jun, c-fos and hsp70mRNAs after folic acid and ischemia-reperfusion injury: effect of antioxidant treatment," Experimental Nephrology, vol. 2, no. 3, pp. 158-165, 1994.

[19] K. Amemiya, Y. Kaneko, T. Muramatsu, M. Shimono, and T. Inoue, "Pulp cell responses during hypoxia and reoxygenation in vitro," European Journal of Oral Sciences, vol. 111, no. 4, pp. 332-338, 2003.

[20] D. W. Leung, G. Cachianes, W. J. Kuang, D. V. Goeddel, and N. Ferrara, "Vascular endothelial growth factor is a secreted angiogenic mitogen," Science, vol. 246, no. 4935, pp. 1306-1309, 1989.

[21] D. J. Dumont, G. H. Fong, M. C. Puri, G. Gradwohl, K. Alitalo, and M. L. Breitman, "Vascularization of the mouse embryo: a study of flk-1, tek, tie, and vascular endothelial growth factor expression during development," Developmental Dynamics, vol. 203, no. 1, pp. 80-92, 1995.

[22] H. F. Dvorak, M. Detmar, K. P. Claffey, J. A. Nagy, L. van de Water, and D. R. Senger, "Vascular permeability factor/vascular endothelial growth factor: an important mediator of angiogenesis in malignancy and inflammation," International Archives of Allergy and Immunology, vol. 107, no. 1-3, pp. 233-235, 1995.

[23] D. W. Dvorak, G. Abbas, T. Ali, S. Stevenson, and D. B. Welling, "Repair of chronic tympanic membrane perforations with long-term epidermal growth factor," Laryngoscope, vol. 105, no. 12, pp. 1300-1304, 1995.

[24] J. Sodek, B. Ganss, and M. D. McKee, “Osteopontin,” Critical Reviews in Oral Biology and Medicine, vol. 11, no. 3, pp. 279-303, 2000.

[25] N. Matsuda, K. Yokoyama, S. Takeshita, and M. Watanabe, "Role of epidermal growth factor and its receptor in mechanical stress-induced differentiation of human periodontal ligament cells in vitro," Archives of Oral Biology, vol. 43, no. 12, pp. 987-997, 1998.

[26] S. Ozaki, S. Kaneko, K. A. Podyma-Inoue, M. Yanagishita, and K. Soma, "Modulation of extracellular matrix synthesis and alkaline phosphatase activity of periodontal ligament cells by mechanical stress," Journal of Periodontal Research, vol. 40, no. 2, pp. 110-117, 2005.

[27] A. L. Bolcato-Bellemin, R. Elkaim, A. Abehsera, J. L. Fausser, Y. Haikel, and H. Tenenbaum, "Expression of mRNAs encoding for alpha and beta integrin subunits, MMPs, and TIMPs in stretched human periodontal ligament and gingival fibroblasts," Journal of Dental Research, vol. 79, no. 9, pp. 1712-1716, 2000.

[28] D. M. Brunette, "Mechanical stretching increases the number of epithelial cells synthesizing DNA in culture," Journal of Cell Science, vol. 69, pp. 35-45, 1984.

[29] R. C. Buck, "Reorientation response of cells to repeated stretch and recoil of the substratum," Experimental Cell Research, vol. 127, no. 2, pp. 470-474, 1980.

[30] P. C. Dartsch and H. Hammerle, "Orientation response of arterial smooth muscle cells to mechanical stimulation," European Journal of Cell Biology, vol. 41, no. 2, pp. 339-346, 1986.

[31] T. C. Tsang, "New model for $70 \mathrm{kDa}$ heat-shock proteins' potential mechanisms of function," FEBS Letters, vol. 323, no. 1-2, pp. 1-3, 1993.

[32] A. E. Kabakov and V. L. Gabai, "Protein aggregation as primary and characteristic cell reaction to various stresses," Experientia, vol. 49, no. 8, pp. 706-710, 1993.
[33] M. A. Peake, L. M. Cooling, J. L. Magnay, P. M. Thomas, and A. J. EL Haj, "Cellular responses to mechanical stress selected contribution: regulatory pathways involved in mechanical induction of c-fos gene expression in bone cells," Journal of Applied Physiology, vol. 89, pp. 2498-2507, 2000.

[34] C. Neidlinger-Wilke, H.-J. Wilke, and L. Claes, "Cyclic stretching of human osteoblasts affects proliferation and metabolism: a new experimental method and its application," Journal of Orthopaedic Research, vol. 12, no. 1, pp. 70-78, 1994.

[35] H. M. Beere, "Stressed to death: regulation of apoptotic signaling pathways by the heat shock proteins," Science, vol. 91, p. 31, 2001.

[36] H. M. Beere, B. B. Wolf, K. Cain, et al., "Heat-shock protein 70 inhibits apoptosis by preventing recruitment of procaspase- 9 to the Apaf-1 apoptosome," Nature Cell Biology, vol. 2, no. 8, pp. $469-475,2000$.

[37] M. Tsuruoka, K. Ishizaki, K. Sakurai, K. Matsuzaka, and T. Inoue, "Morphological and molecular changes in denturesupporting tissues under persistent mechanical stress in rats," Journal of Oral Rehabilitation, vol. 35, no. 12, pp. 889-897, 2008.

[38] M. Ohshima, Y. Yamaguchi, P. Mickey, Y. Abiko, and K. Otsuka, "In vitro characterization of the cytokine profile of the epithelial cell rests of Malassez," Journal of Periodontology, vol. 79, no. 5, pp. 912-919, 2008.

[39] J. C. Rincon, Y. Xiao, W. G. Young, and P. M. Bartold, "Production of osteopontin by cultured porcine epithelial cell rests of Malassez," Journal of Periodontal Research, vol. 40, no. 5, pp. 417-426, 2005.

[40] N. Mizuno, H. Shiba, Y. Mouri, et al., "Characterization of epithelial cells derived from periodontal ligament by gene expression patterns of bone-related and enamel proteins," Cell Biology International, vol. 29, no. 2, pp. 111-117, 2005.

[41] N. Shijubo, T. Uede, S. Kon, M. Nagata, and S. Abe, "Vascular endothelial growth factor and osteopontin in tumor biology," Critical Reviews in Oncogenesis, vol. 11, no. 2, pp. 135-146, 2000. 


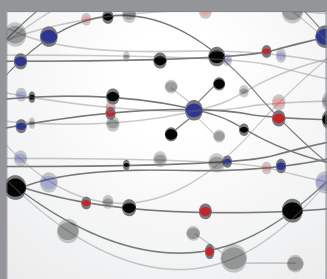

The Scientific World Journal
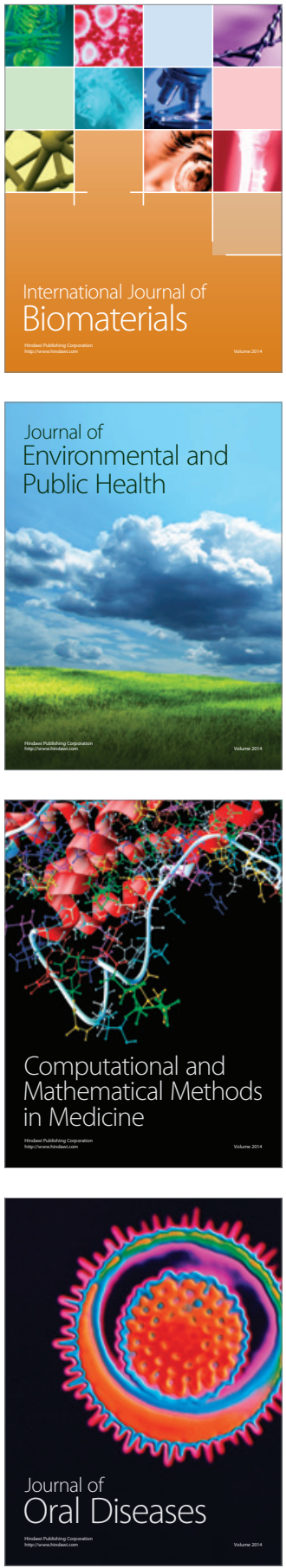
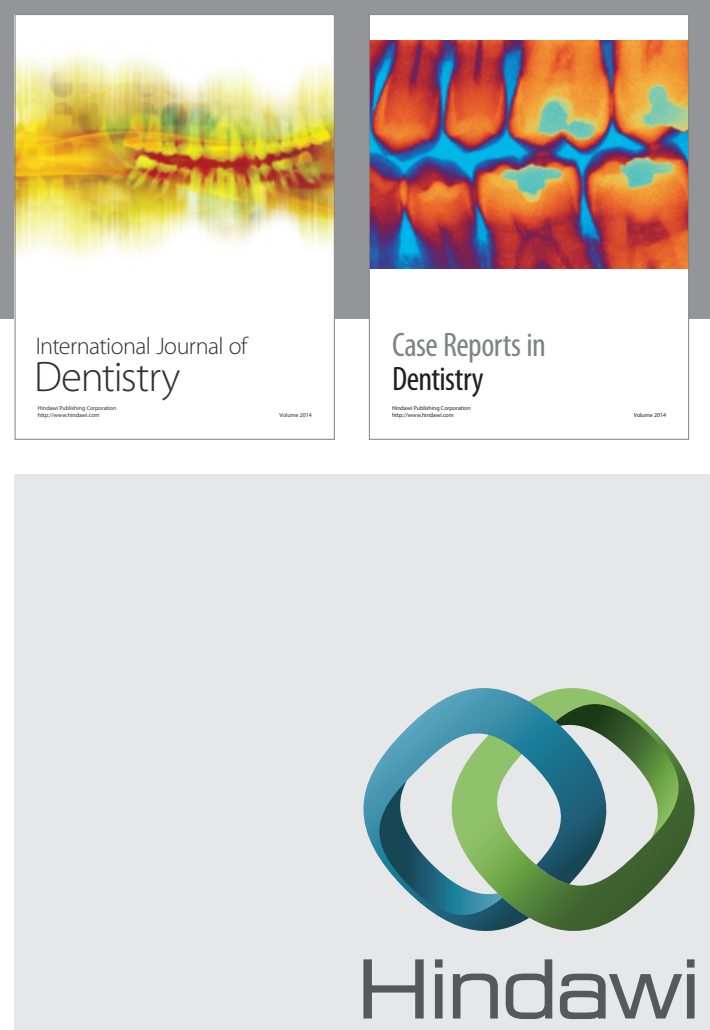

Submit your manuscripts at

http://www.hindawi.com
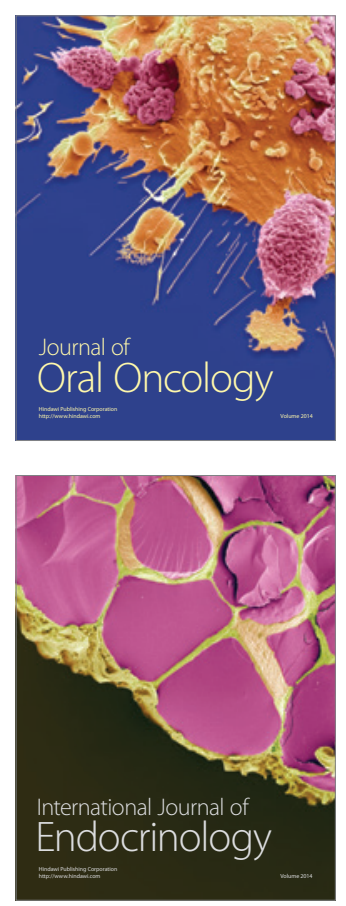
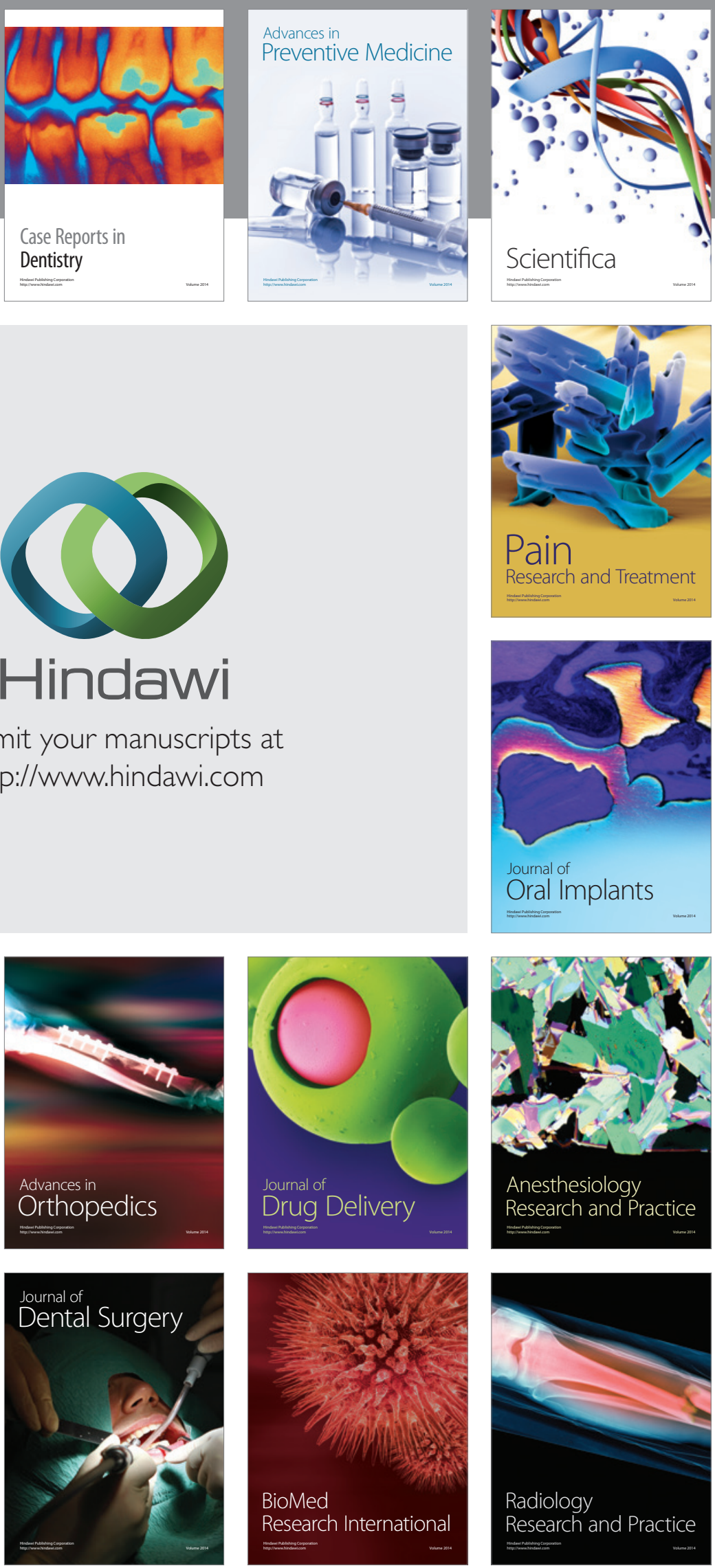